\title{
Effects of epicatechin, a crosslinking agent, on human dental pulp cells cultured in collagen scaffolds
}

\author{
Eun-Su LIM'1 Myung-Jin LIM', Kyung-San MIN'1,2, Young-Sun KWON'1', Yun-Chan HWANG ${ }^{3}$, Mi-Kyung YU', Chan-Ui \\ HONG $^{4}$, Kwang-Won LEE ${ }^{1,2}$
}

\footnotetext{
1- Department of Conservative Dentistry, School of Dentistry and Institute of Oral Bioscience, Chonbuk National University, Jeonju, Korea.

2- Research Institute of Clinical Medicine of Chonbuk National University, Biomedical Research Institute of Chonbuk National University Hospital, Jeonju, Korea.

3- Department of Conservative Dentistry, School of Dentistry, Chonnam National University, Gwangju, Korea.

4- Department of Conservative Dentistry, School of Dentistry, Dankook University, Cheonan, Korea.
}

Corresponding address: Kyung-San Min - Professor of the Department of Conservative Dentistry - School of Dentistry, Chonbuk National University - 567 Baekje-daero - Deokjin-gu - Jeonju-si - Jeollabuk-do - Korea - 561-756 - Phone: +82 632704982 - Fax: +82 632704004 - e-mail: endomin@gmail.com

Submitted: September 1, 2015 - Modification: November 24, 2015 - Accepted: January 4, 2016

$\mathrm{O}$ bjective: The purpose of this study was to investigate the biological effects of epicatechin (ECN), a crosslinking agent, on human dental pulp cells (hDPCs) cultured in collagen scaffolds. Material and Method: To evaluate the effects of ECN on the proliferation of hDPCs, cell counting was performed using optical and fluorescent microscopy. Measurements of alkaline phosphatase (ALP) activity, alizarin red staining, and real-time polymerase chain reactions were performed to assess odontogenic differentiation. The compressive strength and setting time of collagen scaffolds containing ECN were measured. Differential scanning calorimetry was performed to analyze the thermal behavior of collagen in the presence of ECN. Results: Epicatechin increased ALP activity, mineralized nodule formation, and the mRNA expression of dentin sialophosphoprotein (DSPP), a specific odontogenic-related marker. Furthermore, ECN upregulated the expression of DSPP in hDPCs cultured in collagen scaffolds. Epicatechin activated the extracellular signal-regulated kinase (ERK) and the treatment with an ERK inhibitor (U0126) blocked the expression of DSPP. The compressive strength was increased and the setting time was shortened in a dose-dependent manner. The number of cells cultured in the ECN-treated collagen scaffolds was significantly increased compared to the cells in the untreated control group. Conclusions: Our results revealed that ECN promoted the proliferation and differentiation of hDPCs. Furthermore, the differentiation was regulated by the ERK signaling pathway. Changes in mechanical properties are related to cell fate, including proliferation and differentiation. Therefore, our study suggests the ECN treatment might be desirable for dentin-pulp complex regeneration.

Keywords: Catechin. Collagen. Differentiation. Dental pulp. Scaffold.

\section{NTRODUCTI ON}

Apexification has been used to treat pulp pathosis in immature teeth with open apices. The disadvantage of apexification is that it neither allows the root wall to thicken nor continuously develops the immature $\operatorname{root}^{19}$. To overcome these disadvantages of apexification, regenerative endodontic therapy was introduced ${ }^{10}$. In this approach, a blood clot serves as a scaffold for the growth and differentiation of stem cells. However, there have been many controversies over regenerative endodontic therapy regarding the nature of the regenerated tissue and its predictability ${ }^{22}$. Meanwhile, as biotechnology has developed there have been many attempts to regenerate pulp-dentin complexes using tissue engineering concepts.

Tissue engineering treatments involve stem cells, scaffolds, and suitable growth factors. Among these, three-dimensional scaffolds offer an adhesive substrate for cultured cells and a physical support to form organs. Since collagen is an element present in all tissues and non-toxic materials, it is a common 
and useful scaffold. In particular, the dentin organic matrix is described as a macromolecular complex of which approximately $90 \%$ is composed of collagen ${ }^{6}$. In addition, collagen is available as a hydrogel scaffold, which can be applied in a small and narrow prepared root canal space. This feature of collagen hydrogels suggests the appropriateness for its use in regenerative endodontic therapy ${ }^{18}$. In this respect, a collagen hydrogel scaffold is considered useful for the regeneration of the pulpdentin complex. Although collagen is suitable for regenerative therapy, it has disadvantages such as weak physical properties and rapid biodegradation. Therefore, various crosslinking agents have been used to overcome these shortcomings; one of them is glutaraldehyde. Glutaraldehyde is cheaper and has a quicker reaction time than collagen but causes severe toxicity at the site of application because of the release of unreacted aldehydes ${ }^{9}$. Thus, less toxic crosslinking agents must be investigated.

Epicatechin (ECN) is a naturally produced flavanol found in cacao and green tea, and has been orally ingested safely by humans for many centuries (Figure $1 \mathrm{~A})^{17}$. Epicatechin and its derivatives are known to have great potential for anti-inflammatory ${ }^{7}$ and anti-cancer effects ${ }^{4,8}$. Furthermore, they are also used as crosslinking agents ${ }^{3,15}$. However, there have been no studies regarding the effect of ECN on human dental pulp cells (hDPCs) or on the cells cultured in a collagen hydrogel scaffold. Therefore, the aim of this study was to investigate the effects of ECN on the proliferation and differentiation of hDPCs and the physical properties of collagen, as well as its suitability as a scaffold for regenerative endodontic therapy. We also briefly examined the role of extracellular signal-regulated kinase (ERK) as a mediator of ECN-induced differentiation in hDPCs.

\section{MATERI AL AND METHODS}

\section{Primary culture of hDPCs}

Freshly extracted human third molars were obtained from the Chonbuk National University Dental Hospital in Jeonju, Korea. The teeth were sectioned, and dental pulp tissue was removed aseptically. The tissue was minced into small fragments, which were then cultured in minimal essential medium-a (HyClone Laboratories, Logan, UT, USA) containing $10 \%$ of fetal bovine serum (Invitrogen, Carlsbad, CA, USA) along with 100 $\mathrm{U} / \mathrm{mL}$ of penicillin and of $100 \mathrm{U} / \mathrm{mL}$ streptomycin (Invitrogen). Cultures were incubated at $37^{\circ} \mathrm{C}$ in a humidified atmosphere of $5 \% \mathrm{CO}_{2}$ and $95 \%$ air. Cell cultures between the third and fifth passages were used in this study. All experimental procedures were approved by the Institutional Review Board.

\section{Cell viability tests}

The hDPCs were seeded in 24-well culture plates at a density of $2 \times 10^{4}$ cells per well and incubated in growth medium for $24 \mathrm{~h}$. Then, the cells were exposed to various concentrations $(0,0.01,0.05$, 0.1 , and $1 \mathrm{mM}$ ) of ECN (Sigma-Aldrich, St. Louis, MO, USA) for up to 14 days. Cell viability was evaluated using the 3-(4,5-dimethylthiazol-2-yl)2,5-diphenyltetrazolium bromide (MTT) assay. Briefly, $200 \mathrm{~mL}$ of MTT solution $(0.5 \mathrm{mg} / \mathrm{mL}$ in PBS) was added to each well and the wells were incubated for $2 \mathrm{~h}$. Subsequently, $200 \mathrm{~mL}$ of dimethyl sulfoxide (DMSO; Amresco, Solon, OH, USA) was added to each well. The plates were shaken until the crystals had dissolved and the solution in each well was transferred to a 96-well tissue culture plate. Reduced MTT was then measured spectrophotometrically at $540 \mathrm{~nm}$ in a microplate reader (SPECTROstar Nano, BMG Labtech, Ortenberg, Germany).

\section{Alkaline phosphatase (ALP) activity}

The hDPCs were seeded in six-well culture plates at a density of $2 \times 10^{4}$ cells per well and were incubated for $24 \mathrm{~h}$ for the initial attachment. After the cells were incubated for one, three, or seven days in the presence of various concentrations of $\operatorname{ECN}(0,0.01,0.05$ or $0.1 \mathrm{mM})$, they were scraped into cold PBS (HyClone Laboratories). The ALP activity in the supernatant was determined according to the Sensolyte pNPP ALP Assay Kit (AnaSpec Inc, Fremont, CA, USA) using a modified method established in a previous study ${ }^{16}$. The absorbance was measured at $405 \mathrm{~nm}$ using a microplate reader (SPECTROstar Nano).

\section{Alizarin red S staining}

The hDPCs were distributed in a 24-well culture plate at a density of $2 \times 10^{4}$ and cultured for 24 h. Then, the cells were treated with different concentrations of ECN for the duration of the experiment. After the hDPCs were cultured for 14,21 , and 28 days, the cells were washed with PBS and reacted with $40 \mathrm{mmol} / \mathrm{L}$ of alizarin red S solution (Sigma-Aldrich) adjusted to a $\mathrm{pH}$ of 4.2 with ammonium hydroxide. After being washed with distilled water, the samples were reacted with $10 \%$ cetylpyridinium chloride solution (SigmaAldrich) at room temperature for $15 \mathrm{~min}$ to dissolve the stain. The contents from each well were transferred to a 96-well plate and were measured spectrophotometrically in a microplate reader (SPECTROstar Nano).

Gene expression analysis of real-time polymerase chain reaction ( $P C R$ )

After exposure to ECN for 7 days, the hDPCs cultured in collagen were lysed directly in the plates using $1.0 \mathrm{~mL}$ Trizol reagent (Invitrogen). After 
chloroform extraction, the total RNA was recovered from the aqueous phase and precipitated with isopropanol and RNAase-free distilled water. Then, reverse transcription of RNA was performed using a Superscript First-Strand Synthesis kit (I nvitrogen). Thereafter, the reverse transcriptase-generated first-strand DNA was amplified.

Reactions for real-time PCR were prepared as follows: $12.5 \mu \mathrm{l}$ of QuantiTect SYBR Green PCR master mix (Qiagen, Hilden, NRW, Germany), 2.5 $\mu \mathrm{l}$ of each primer, and $8 \mu \mathrm{l}$ of distilled water were used per $25-\mu$ l reaction volume. Two microliters of template were added to each of the tubes. Samples were always measured in triplicate, while standard curve samples were only single measured as triplicate dilution series were prepared. Primers used for real-time PCR were purchased from Qiagen: dentin sialophosphoprotein (DSPP; catalog \#QT00061887), dentin matrix protein 1 (DMP1; catalog \#QT00022078), osteonectin (ON; catalog \#QT00018620), and glyceraldehyde 3-phosphate dehydrogenase (GAPDH; catalog \#QT00079247). Real-time PCR was performed in a Rotor-Gene Q real-time PCR machine (Qiagen) in the 72-well rotor. The thermocycling program for real-time PCR was as follows: $5 \mathrm{~min}$ at $95^{\circ} \mathrm{C}$ for the initial denaturation, followed by 40 cycles of $10 \mathrm{~s}$ at $95^{\circ} \mathrm{C}$, $10 \mathrm{~s}$ at $60^{\circ} \mathrm{C}$, and $20 \mathrm{~s}$ at $72^{\circ} \mathrm{C}$. Finally, a melting curve ranging from 75 to $95^{\circ} \mathrm{C}$ was performed with increments of $1^{\circ} \mathrm{C}$.

\section{Western blotting}

The hDPCs were distributed in $60 \mathrm{~mm}$ plates with consistent density. Cells were then incubated in the presence of ECN $(0.1 \mathrm{mM})$ for $0,10,30,60$, or $120 \mathrm{~min}$. Cells were washed using $1 \times$ PBS (Hyclone Laboratories) and were scraped using the cell scraper. A protein lysis buffer was used for $10 \mathrm{~min}$ on ice to solubilize the cells. The resolved solutions were centrifuged at 13,000 rpm for $10 \mathrm{~min}$, and the supernatants were transferred to other tubes. Protein concentrations were measured using the Bradford reagent (Bio-Rad Laboratories, Hercules, CA, USA). Analyzed proteins were separated with $9 \%$ sodium dodecyl sulfate-polyacrylamide gel electrophoresis and transferred to nitrocellulose membranes (Protran; Whatman, Dassel, Lower Saxony, NI, Germany). Membranes were blocked with $5 \%$ skim milk in Tris-buffered saline and Tween 20 for $30 \mathrm{~min}$ at room temperature. After being washed three times, the membranes were incubated overnight at $4^{\circ} \mathrm{C}$ with primary antibodies against $p$-ERK (Cell Signaling Technology, Danvers, MA, USA) or ERK (Cell Signaling Technology), followed by incubation with horseradish peroxidaseconjugated secondary antibodies. Separated proteins were detected using the ECL Western Blotting Luminol agent (Santa Cruz Biotechnology,
Santa Cruz, CA, USA).

Effect of ERK inhibition on expression of odontoblastic/ osteoblastic markers

The hDPCs were cultured in $60 \mathrm{~mm}$ plates for 7 days with or without $5 \mu \mathrm{M}$ U0126 (Promega, Madison, WI , USA), an inhibitor of $p$-ERK. Cells were pre-incubated with U0126 for $1 \mathrm{~h}$ before treatment with ECN. The inhibitor was dissolved in DMSO, and control cells were pre-incubated with equivalent amounts of DMSO alone.

\section{Preparation of collagen scaffolds}

The collagen scaffold was prepared by using the method described previously ${ }^{13}$. Briefly, a purified bovine collagen solution (PurCol; Advanced BioMatrix Inc., Tucson, AZ, USA) and $10 \times$ PBS were mixed in an 8: 1 ratio and adjusted to a pH of 7.4 with $1 \mathrm{~mL}$ of sodium hydroxide ( $1 \mathrm{M}$ ) (Bio Basic Canada Inc., Markham, ON, Canada). The mixture was incubated at $37^{\circ} \mathrm{C}$ for $2 \mathrm{~h}$ further.

\section{Cell number measurement}

Six-well culture plates were coated with $1.5 \mathrm{~mL}$ of collagen solution with or without $\operatorname{ECN~}(n=10)$. After collagen gelation, the hDPCs $\left(1 \times 10^{5}\right)$ were seeded onto the specimens and cultured for 3 days. The cells cultured in half of the plates were stained with 4',6-diamidino-2-phenylindole (DAPI) (Invitrogen). Then, the cell numbers were determined under an optical (Leica, Solms, Hessen, $H E$, Germany) $(n=5)$ or fluorescent microscope (Carl Zeiss, Jena, Thuringia, Germany) $(n=5)$ by two calibrated examiners who did not have any information regarding the experiment. Briefly, a rectangle was inscribed in a round six-well plate and divided into nine equal areas. Then, three images were acquired from each area, and the number of cells [mean \pm standard deviation (SD)] in each area was counted by the examiners.

\section{Setting time}

The setting times of the collagen scaffolds were measured using the tilting method described previously ${ }^{18}$. Purified bovine collagen solution was mixed with $10 \times$ PBS at a ratio of $8: 1$ (volume). The $\mathrm{pH}$ was adjusted to 7.4 by adding $1 \mathrm{~mL}$ of sodium hydroxide ( 1 M) (Bio Basic Canada Inc., Markham, ON, Canada). Then, the mixture was treated with 0 or $1 \mu \mathrm{M}$ of ECN and inserted into an Eppendorf tube. We considered the hydrogel collagen to be set if it did not flow when the tube was tilted $45^{\circ} \mathrm{C}$. The samples $(n=5)$ were tested just before their anticipated setting times and at $30 \mathrm{~s}$ intervals.

\section{Compressive strength}

The compressive strength was evaluated using the method described previously ${ }^{13}$. Round 
polymerized collagen samples ( $1 \mathrm{~mm} \times 2.5 \mathrm{~mm}$ ) treated with $\operatorname{ECN}(0,0.01,0.05$, or $0.1 \mathrm{mM})$ were prepared $(n=5)$. Then, their compressive strengths were measured using a universal testing machine (TMS-Pro; Food Technology Corp., Sterling, VA, USA) with a $0.5-\mathrm{N}$ load cell moving at a crosshead speed of $1 \mathrm{~mm} / \mathrm{min}$.

\section{Differential scanning calorimetry (DSC)}

A differential scanning calorimeter (Q20; TA Instruments, New Castle, DE, USA) was used to determine the thermal behavior of the crosslinked collagen scaffolds. The collagen scaffolds were examined under wet conditions. The heating was carried out at $10^{\circ} \mathrm{C} / \mathrm{min}$ under nitrogen air in a temperature range from 30 to $330^{\circ} \mathrm{C}$. The denaturation temperature was determined as the peak value of the corresponding endothermic phenomena and the denaturation enthalpy was calculated as the area of the peak regarding the weight of collagen.

\section{Statistical analysis}

Statistical analysis was performed using the Student's t-test or the one-way analysis of variance (ANOVA), followed by a multiple-comparison test (Tukey's test). A p-value smaller than 0.05 was considered statistically significant.

\section{RESULTS}

\section{Cell viability test}

We evaluated the effects of ECN on cell viability using an MTT assay. As shown in Figure 1B, hDPCs treated with $0.01,0.05$, or $0.1 \mathrm{mM}$ of ECN did not show any statistically significant difference in viability compared to the cells in the control group $(p>0.05)$. However, the cells treated with $1 \mathrm{mM}$ of ECN showed significantly lower viability $(p<0.05)$. Based on this result, we selected the concentration of $0.1 \mathrm{mM}$ for use in this study.

\section{Alkaline phosphatase (ALP) activity}

Alkaline phosphatase activity was measured as an early marker of odontogenic differentiation of hDPCs. There were no significant differences between the ECN-treated cells and control cells through day 3 ( $p>0.05)$. However, on day 7 , the ALP activity increased in the cells treated with 0.1 $\mathrm{mM}$ of ECN $(p<0.05)$ (Figure $1 C)$.

\section{Alizarin red S staining}

We evaluated the mineralization of the extracellular matrix using alizarin red $\mathrm{S}$ staining. The formation of mineralized nodules in the ECNtreated cells was significantly higher than that in the control group cells on day 28 ( $p<0.05$; Figure 1D).
Expression of odontogenic markers in hDPCs per se

Dentin sialophosphoprotein, DMP1, and ON served as odontogenic markers. As shown in Figure $1 \mathrm{E}$, the expression of DSPP mRNA increased in the presence of $0.1 \mathrm{mM}$ of ECN on day 7 .

\section{Effect of the ERK inhibitor on ECN-induced odontogenic marker expression}

Epicatechin $(0.1 \mathrm{mM})$ caused the accumulation of $\mathrm{p}$-ERK in hDPCs within $30 \mathrm{~min}$ of treatment (Figure 1F). As shown in Figure 1G, $5 \mu \mathrm{M}$ of U0126 effectively blocked the expression of p-ERK after $30 \mathrm{~min}$. To address whether the ERK pathway influences odontogenic differentiation, real-time PCR was performed on hDPCs treated with $0.1 \mathrm{mM}$ ECN in medium with or without $5 \mu \mathrm{M}$ U0126 for 7 days. The results showed the expression of DSPP decreased significantly with U0126 treatment in ECN-treated hDPCs (Figure $1 \mathrm{H}$ ).

\section{Cell number measurement}

Cell numbers were measured under optical (Figures 2A and B) and fluorescence microscopes (Figures $2 \mathrm{C}$ and $\mathrm{D}$ ). The number of cells in the ECNtreated crosslinked group was significantly higher than that of the untreated control group $(p<0.05$; Figure 2E).

Expression of odontogenic markers in hDPCs cultured in collagen scaffolds

We assessed whether the collagen crosslinking using ECN promoted the expression of odontogenic markers in hDPCs cultured in collagen scaffolds. As shown in Figure 2F, the expression of DSPP mRNA was higher in the cells cultured in the ECN-treated collagen compared to the cells in the control group $(p<0.05)$.

\section{Setting time and compressive strength}

The setting times of the ECN-treated collagens were significantly lower than those of the untreated controls $(p<0.05$; Figure $3 A)$. The compressive strength of the cross-linked collagen increased in a dose-dependent manner. However, only the 0.1 mM ECN group exhibited a significant difference in compressive strength compared to the untreated control group $(p<0.05$; Figure $3 B)$.

\section{Differential scanning calorimetry}

Differential scanning calorimetry studies were undertaken to evaluate the thermal behavior of collagen scaffolds, and the thermograms are displayed in Figure $3 \mathrm{C}$. The thermogram of the uncrosslinked collagen exhibited an endothermic sharp peak at $75.41^{\circ} \mathrm{C}$ due to its denaturation, whereas the collagen crosslinked with $0.1 \mu \mathrm{M}$ ECN exhibited a peak at $112.32^{\circ} \mathrm{C}$. 
A<smiles>Oc1cc(O)c2c(c1)O[C@H](c1ccc(O)c(O)c1)[C@H](O)C2</smiles>
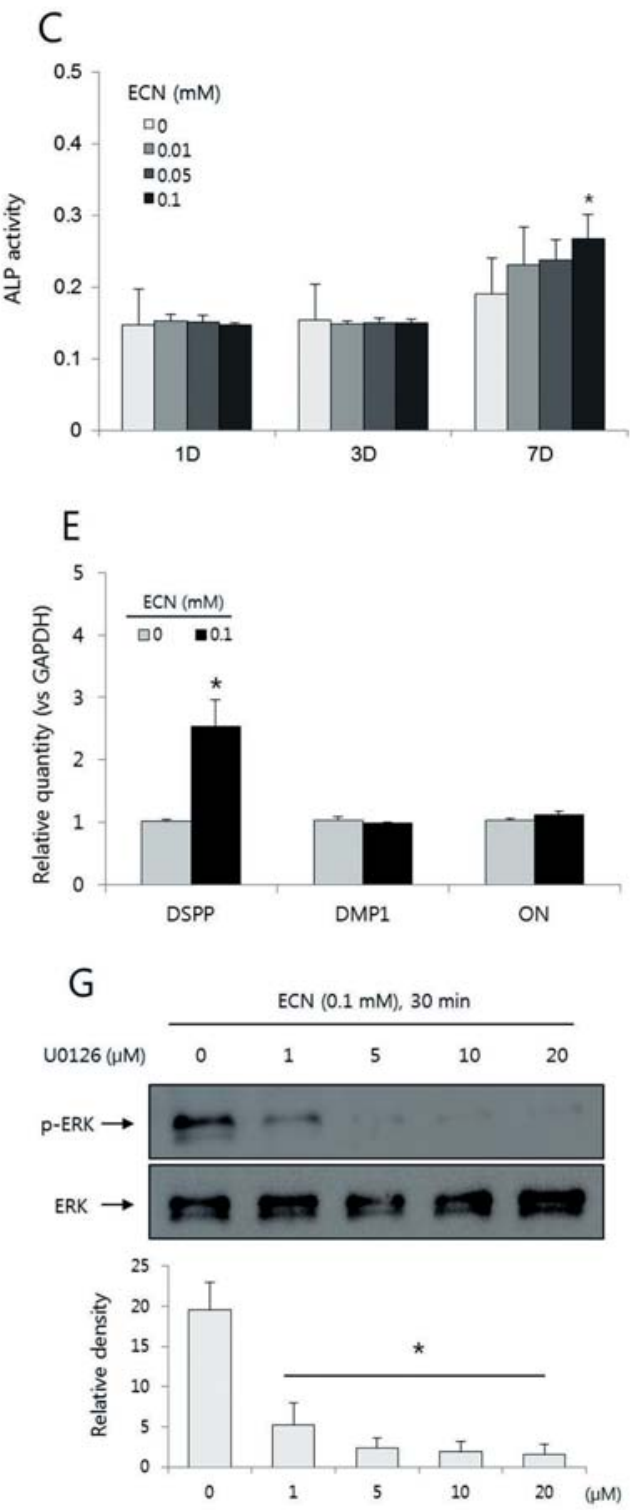

\section{B}
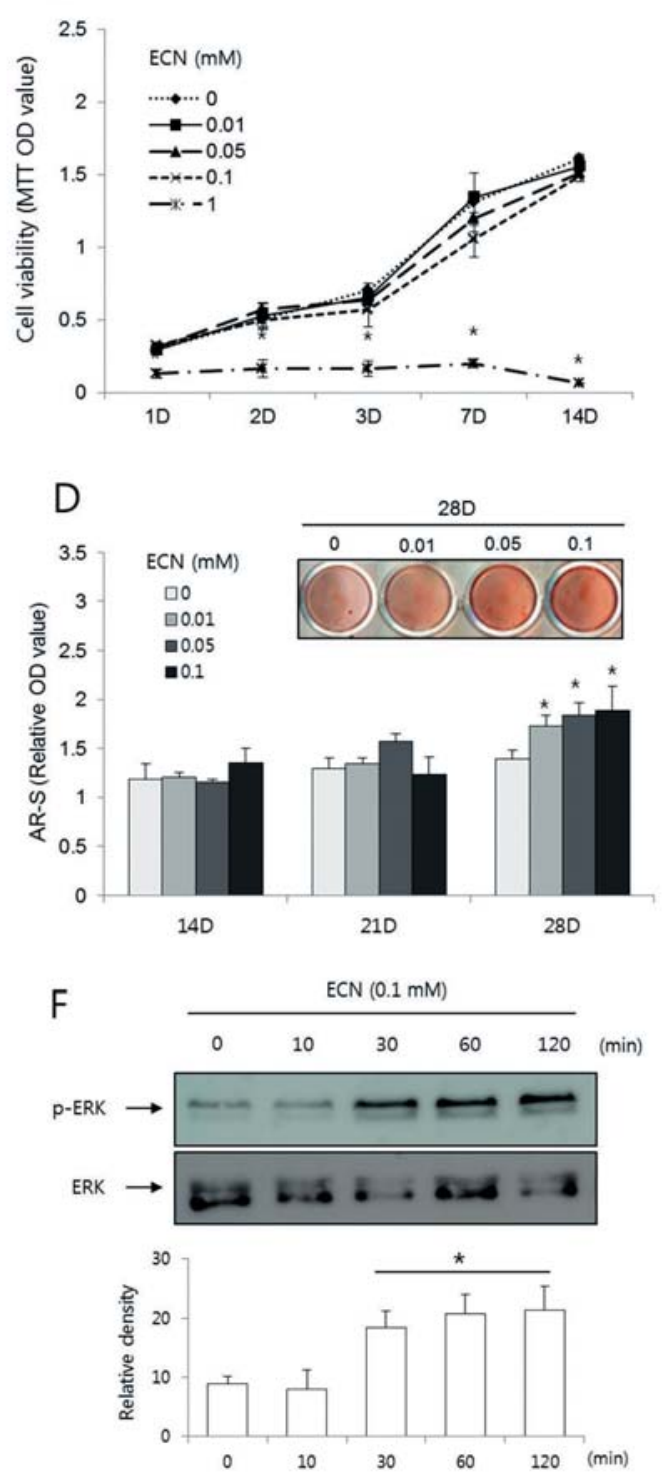

$\mathrm{H}$

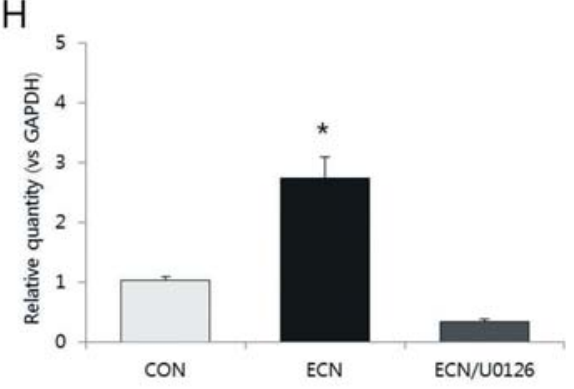

Figure 1- Effects of epicatechin (ECN) on odontogenic differentiation of human dental pulp cells (hDPCs). (A) Chemical structure of ECN. Effects of ECN on cell viability measured by the MTT assay (B), the ALP activity (C), and the formation of calcification nodules (D) in hDPCs. *A significant difference compared with the control was determined at $p<0.05$. $(E)$ Effects of ECN on the expression of DSPP, DMP1, and ON mRNA in hDPCs cultured for 7 days. (F) p-ERK expression increased within 30 minutes. (G) p-ERK expression was effectively blocked by $5 \mu \mathrm{M}$ of $\mathrm{U} 0126$. (H) The expression level of DSPP mRNA induced by ECN (0.1 mM) was blocked by U0126 ( $5 \mu \mathrm{M})$ after 7 days. The relative levels of gene expression were normalized against GAPDH, and the control level was set as 1.0. *Significant difference compared with the control $(\mathrm{p}<0.05)$ 

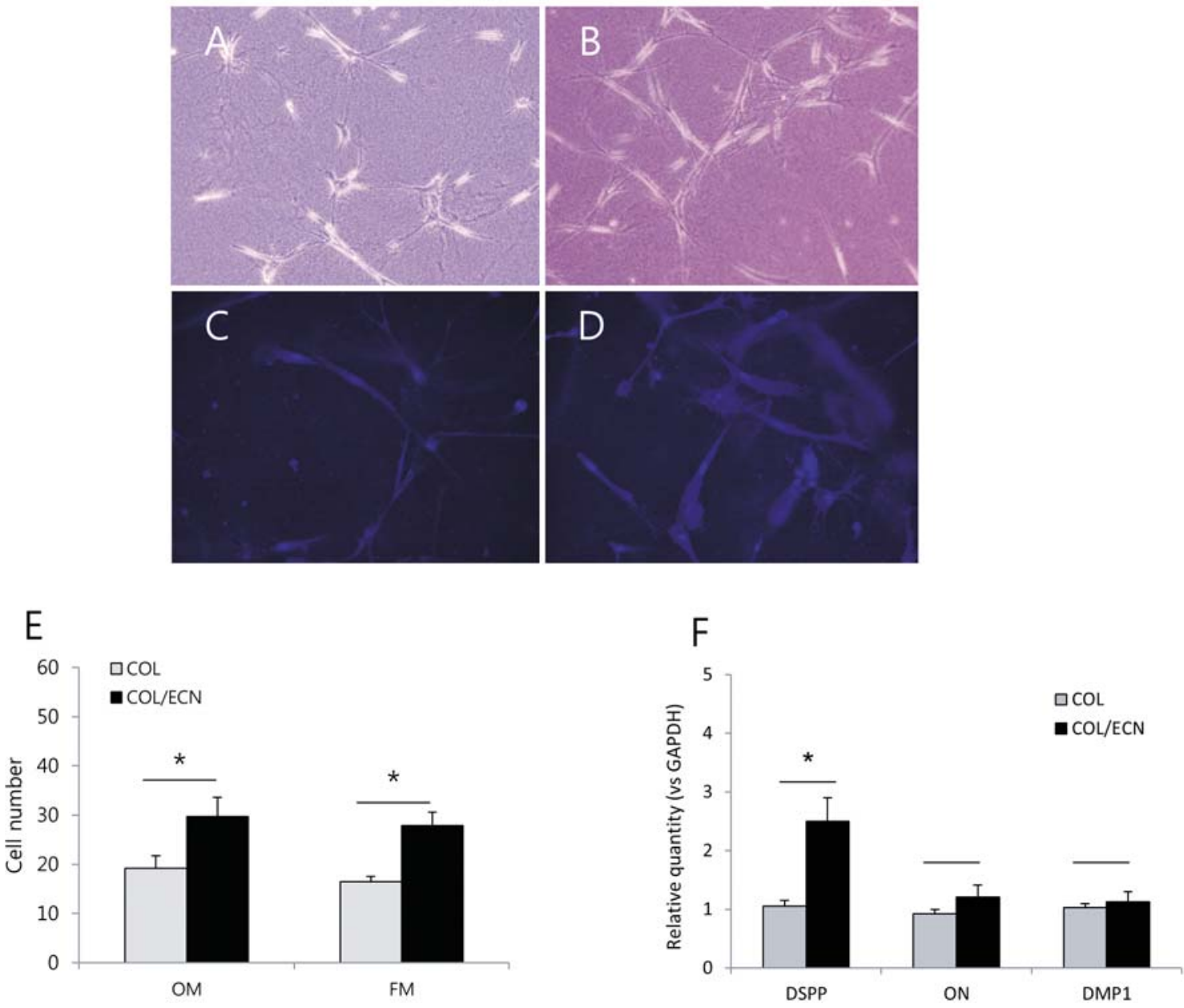

Figure 2- Proliferation analysis of human dental pulp cells (hDPCs) cultured in collagen scaffolds. Optical microscopic views of hDPCs cultured in untreated $(A)$ and epicatechin $(E C N)$-treated collagen matrices $(B)(\times 200)$. Representative fluorescence microscopy images $(\times 200)$ showing hDPCs (stained with DAPI) growing in the collagen scaffold after 3 days of culture in the absence (C) or presence (D) of epicatechin. (E) Effects of ECN-induced crosslinking on proliferation of hDPCs cultured in collagen; OM: optical microscope, FM: fluorescence microscope. (F) The effects of ECN on the expression of DSPP, DMP1, and ON mRNAs in hDPCs cultured in collagen scaffold for 7 days. The relative gene expression levels were normalized against GAPDH and the control was set as 1.0. ${ }^{*}$ Significant difference compared with control $(p<0.05)$

\section{SCUSSI ON}

Crosslinking of collagen scaffolds is considered a favorable technique because it enhances many beneficial properties of the scaffold and may provide some advantages concerning cell growth and fate. Recently, several naturopathic medicines are gaining attention for collagen crosslinking because of their low cytotoxicity compared to glutaraldehyde 2,3,5,11,13. Among these, ECN and its derivatives have been reported for having various pharmacologic functions including anti-inflammatory and anti-cancer effects. However, there was no previous information regarding the odontogenic effect of ECN in pulp cells for dentin-pulp complex engineering. Therefore, we investigated whether ECN, a collagen cross-linking agent, promoted odontogenic differentiation of
hDPCs.

We examined the effects of $E C N$ on the odontogenic differentiation of hDPCs in this study. Treatment with $0.1 \mathrm{mM}$ of ECN increased ALP activity, calcium nodule formation, and expression of DSPP mRNA, which is considered a specific biochemical marker of functional odontoblasts (Figure 1C-E). ${ }^{1}$ Zeng, et al. ${ }^{23}$ (2014) demonstrated the ECN promoted osteoblast differentiation and might have beneficial effects on bone loss prevention. Wei, et al. ${ }^{21}$ (2011) also showed the catechin stimulates osteogenesis in mesenchymal stem cells. Furthermore, Kwon, et al. ${ }^{13}$ (2015) recently introduced genipin, another collagen crosslinking agent, which has stimulatory effects on odontogenic differentiation, and suggested that cross-linking of collagen may be advantageous for pulp-denin complex regeneration. In this respect, 
A

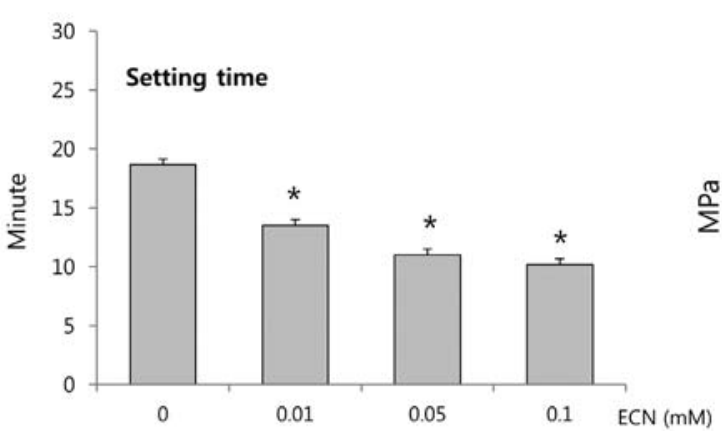

B

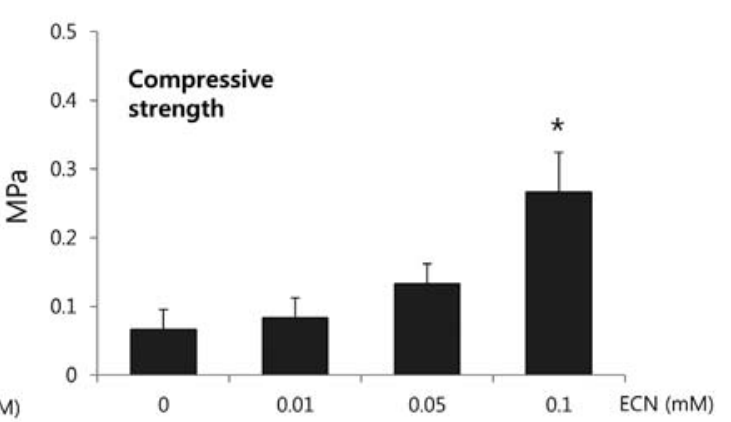

C

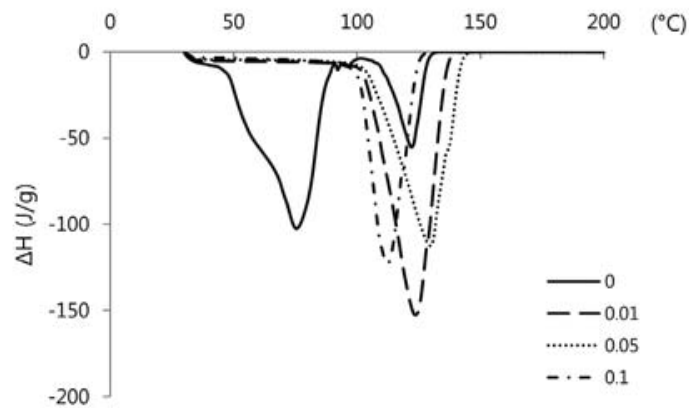

\begin{tabular}{ccc}
\hline ECN $(\mathbf{m M})$ & $\mathrm{T}_{\mathrm{D}}\left({ }^{\circ} \mathrm{C}\right)$ & $\Delta \mathrm{H}(\mathrm{J} / \mathrm{g})$ \\
\hline 0 & 75.41 & 1533 \\
0.01 & 123.62 & 2102 \\
0.05 & 130.39 & 2149 \\
0.1 & 112.32 & 2048 \\
\hline
\end{tabular}

Figure 3- Setting time (A) and compressive strength $(B)$ of collagen in the presence of various concentrations of epicatechin $(E C N)$. (C) Differential scanning calorimetric curves of uncrosslinked and crosslinked collagen scaffolds. *Significant difference compared with control $(p<0.05)$

the application of ECN might be beneficial for the odontogenic differentiation of hDPCs incorporated in collagen scaffolds for use in regenerative therapy.

Then, we investigated the mechanism of ECN for the differentiation of hDPCs. Since the ERK pathway has been reported as an important mechanism for odontogenic differentiation in hDPCs ${ }^{12-14}$, we postulated that ERK might play a significant role in ECN-induced odontogenic differentiation. As shown in Figure 1F, $0.1 \mathrm{mM}$ of ECN increased the phosphorylation of ERK within 30 min. Furthermore, the blocking of the ERK signaling by U0126, a specific antagonist of ERK, inhibited the expression of DSPP, the odontology-related gene that was upregulated by treatment with ECN (Figure $1 \mathrm{H}$ ). Based on these experimental results, it is reasonable to assume the ERK is a regulator of the ECN-induced odontogenic differentiation of hDPCs.

Next, we evaluated the effect of ECN treatment on the proliferation and differentiation of hDPCs cultured in collagen hydrogel scaffolds. As shown in Figure $2 \mathrm{~A}-\mathrm{E}$, the number of cells in the crosslinked group was statistically higher than that of the uncrosslinked control group. Similar to the aforementioned real-time PCR results, the expression of DSPP was increased in the cells cultured in ECN-treated collagen compared with the cells in untreated collagen (Figure 2F). In this study, two different types of microscopes were used to accurately count the cell numbers. Actually, it is difficult to count cells cultured in collagen scaffolds using optical microscopes because of the overlying collagen. To solve this problem, these cells were stained with DAPI to increase their contrast with the scaffolds. We double-checked the effect of ECN on the proliferation of hDPCs using two different microscopes, and the results were similar. Although the effect of ECN on the differentiation of hDPCs was predictable, the reason for the increasing in cell growth is not clear.

We hypothesized that the quicker setting time and enhanced mechanical properties of the crosslinked collagen contributed to the increased cell proliferation. To verify this hypothesis, setting times and compressive strengths were assessed as mechanical properties. We found the setting time was significantly shortened by treating the collagen with ECN (Figure $3 \mathrm{~A}$ ). The gel phase of hydrogel provides a more suitable environment for cells to attach and grow compared to the sol phase. The faster conversion of collagen hydrogel into the gel phase might provide more opportunities for the cells to attach to the substrate. Furthermore, the compressive strengths of ECN-treated collagen scaffolds were higher than those of the untreated collagen, increasing in a dose-dependent manner (Figure 3B). Mechanical properties are important for maintaining a three-dimensional architecture for cell attachment and migration ${ }^{20}$. Zoldan, et al. ${ }^{24}$ (2011) suggested that mechanical stimuli play an 
important role in stem cell differentiation. As the addition of more crosslinking agents improves the mechanical properties of collagen scaffolds, this might contribute to cell proliferation and differentiation ${ }^{13}$. According to the previous studies and the current results, we suggest that a quicker setting time and increased compressive strength of collagen scaffolds, which were achieved by ECN-induced crosslinking, are beneficial to the proliferation and differentiation of hDPCs.

Lastly, DSC measurement was performed to investigate the thermal behavior of collagen scaffolds. The denaturation point is a physical parameter used to identify the nature of a substance. As shown in DSC results (Figure $3 C$ ), the enthalpy of samples denaturation $(\Delta H)$ was determined by the peak in the DSC curve. The higher melting peak in the crosslinked collagen samples indicates the samples consumed more energy for denaturation than the uncrosslinked collagen samples. Therefore, the application of ECN into the scaffolds stabilizes the substrate based on the molecular interaction between the collagen and ECN, and this stabilization increases cell proliferation along with the aforementioned mechanisms. In addition, this property might be beneficial for preventing the degradation of collagen scaffolds used in future in vivo studies.

\section{CONCLUSI ONS}

Collectively, our results suggest the crosslinking of collagen with ECN positively affects the attachment, proliferation, and differentiation of hDPCs cultured in collagen scaffolds, and that this differentiation is regulated by the ERK signaling pathway. Furthermore, the enhanced physical properties of collagen hydrogel scaffolds induced by the ECN treatment might play important roles in cell fate such as proliferation and differentiation. Therefore, within the limitations of the current study, the crosslinking of collagen with ECN seems to be beneficial for tissue engineering-based regenerative endodontic therapy.

\section{ACKNOWLEDGMENTS}

This paper was supported by the Biomedical Research Institute of the Chonbuk National University Hospital in 2014.

The authors deny any conflicts of interest.

Lim ES and Lim MJ contributed equally to this work as first authors.

Min KS and Lee KW contributed equally to this work as corresponding authors.

\section{REFERENCES}

1- D'Souza RN, Bachman T, Baumgardner KR, Butler WT, Litz $M$. Characterization of cellular responses involved in reparative dentinogenesis in rat molars. J Dent Res. 1995; 74: 702-9.

2- Del Gaudio C, Baiguera S, Boieri M, Mazzanti B, Ribatti D, Bianco $A$, et al. Induction of angiogenesis using VEGF releasing genipin-crosslinked electrospun gelatin mats. Biomaterials. 2013; 34: 7754-65.

3- Fathima NN, Dhathathreyan A, Ramasami T, Krägel J, Miller R. Degree of crosslinking of collagen at interfaces: adhesion and shear rheological indicators. Int J Biol Macromol. 2011;48:67-73. 4- Fraga CG, Oteiza PI. Dietary flavonoids: role of (-)-epicatechin and related procyanidins in cell signaling. Free Radic Biol Med. 2011; 51:813-23.

5- Frohbergh ME, Katsman A, Botta GP, Lazarovici P, Schauer CL, Wegst UG, et al. Electrospun hydroxyapatite-containing chitosan nanofibers crosslinked with genipin for bone tissue engineering. Biomaterials. 2012; 33:9167-78.

6- Goldberg M, Kulkarni AB, Young M, Boskey A. Dentin: structure, composition and mineralization. Front Biosci (Elite Ed). 2011; 3: 711-35.

7- Gutierrez-Salmean G, Ciaraldi TP, Nogueira L, Barboza J, Taub PR, Hogan MC, et al. Effects of (-)-epicatechin on molecular modulators of skeletal muscle growth and differentiation. J Nutr Biochem. 2014; 25: 91-4.

8- Henning SM, Aronson W, Niu Y, Conde F, Lee NH, Seeram NP, et al. Tea polyphenols and theaflavins are present in prostate tissue of humans and mice after green and black tea consumption. J Nutr. 2006; 136: 1839-43.

9- Huang GP, Shanmugasundaram S, Masih P, Pandya D, Amara $S$, Collins $G$, et al. An investigation of common crosslinking agents on the stability of electrospun collagen scaffolds. J Biomed Mater Res A. 2015; 103: 762-71.

10- I waya SI, Ikawa M, Kubota M. Revascularization of an immature permanent tooth with apical periodontitis and sinus tract. Dent Traumatol. 2001; 17: 185-7.

11- Jaiswal M, Gupta A, Agrawa AK, Jassal M, Dinda AK, Koul $\mathrm{V}$. Bi-layer composite dressing of gelatin nanofibrous mat and poly vinyl alcohol hydrogel for drug delivery and wound healing application: in-vitro and in-vivo studies. J Biomed Nanotechnol. 2013; 9: 1495-508.

12- Karanxha L, Park SJ , Son WJ, Nör JE, Min KS. Combined effects of simvastatin and enamel matrix derivative on odontoblastic differentiation of human dental pulp cells. J Endod. 2013; 39: 7682.

13- Kwon YS, Lim ES, Kim HM, Hwang YC, Lee KW, Min KS. Genipin, a cross-linking agent, promotes odontogenic differentiation of human dental pulp cells. J Endod. 2015;41:501-7.

14- Lee YM, Shin SY, Jue SS, Kwon IK, Cho EH, Cho ES, et al. The role of PIN1 on odontogenic and adipogenic differentiation in human dental pulp stem cells. Stem Cells Dev. 2014;23:618-30. 15- Liu Y, Bai X, Li S, Liu Y, Keightley A, Wang Y. Molecular weight and galloylation affect grape seed extract constituents' ability to cross-link dentin collagen in clinically relevant time. Dent Mater. 2015; 31: 814-21.

16- Lowry OH, Roberts NR, Wu ML, Hixon WS, Crawford EJ. The quantitative histochemistry of brain. II. Enzyme measurements. J Biol Chem. 1954;207: 19-37.

17- Nakanishi T, Mukai K, Yumoto H, Hirao K, Hosokawa Y, Matsuo $\mathrm{T}$. Anti-inflammatory effect of catechin on cultured human dental pulp cells affected by bacteria-derived factors. Eur J Oral Sci. 2010; 118: 145-50.

18- Park SJ, Li Z, Hwang IN, Huh KM, Min KS. Glycol chitin-based thermoresponsive hydrogel scaffold supplemented with enamel matrix derivative promotes odontogenic differentiation of human dental pulp cells. J Endod. 2013; 39:1001-7.

19- Petrino JA, Boda KK, Shambarger S, Bowles WR, McClanahan SB. Challenges in regenerative endodontics: a case series. J Endod. 2010; 36: 536-41. 
20- Tierney CM, Haugh MG, Liedl J, Mulcahy F, Hayes B, O'Brien $F J$. The effects of collagen concentration and crosslink density on the biological, structural and mechanical properties of collagenGAG scaffolds for bone tissue engineering. J Mech Behav Biomed Mater. 2009;2:202-9.

21- Wei YJ, Tsai KS, Lin LC, Lee YT, Chi CW, Chang MC, et al. Catechin stimulates osteogenesis by enhancing PP2A activity in human mesenchymal stem cells. Osteoporos Int. 2011;22: 146979.

22- Wigler R, Kaufman AY, Lin S, Steinbock N, Hazan-Molina $\mathrm{H}$ Torneck CD. Revascularization: a treatment for permanent teeth with necrotic pulp and incomplete root. J Endod. 2013;39:319-26.
23- Zeng X, Tian J, Cai K, Wu X, Wang Y, Zheng Y, et al. Promoting osteoblast differentiation by the flavanes from Huangshan Maofeng tea is linked to a reduction of oxidative stress. Phytomedicine. 2014; $21: 217-24$.

24- Zoldan J, Karagiannis ED, Lee CY, Anderson DG, Langer R, Leveberg $S$. The influence of scaffold elasticity on germ layer specification of human embryonic stem cells. Biomaterials. 2011;32:9612-21. 